\title{
CAUSALITY AND STOCHASTIC REALIZATION
}

\author{
LJILJANA PETROVIĆ
}

Received 20 February 2004 and in revised form 3 December 2004

We consider a problem (that follows directly from realization problem) on finding Markovian representations for a given family of Hilbert spaces such that each of these two families provides exactly the same amount of information about some other family of Hilbert spaces.

\section{Introduction}

The study of Granger causality has been mainly preoccupied with time series. We will instead concentrate on continuous time processes. Many systems to which it is natural to apply tests of causality take place in continuous time. For example, this is generally the case within economy.

In the first part of this paper, we give a generalization of a causality relationship " $\mathrm{G}$ is

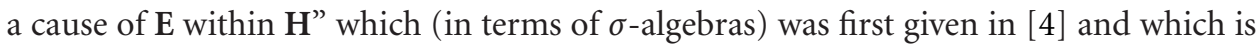
based on Granger's definition of causality [2].

In the second part, we relate concepts of causality to the stochastic realization problem. The approach adopted in this paper is that of [3]. However, since our results do not depend on probability distribution, we deal with arbitrary Hilbert spaces instead of those generated by Gaussian processes.

We suppose that it is known that a stochastic dynamic system (s.d.s.) $S_{1}$ with known outputs $\mathbf{H}$ causes, in a certain sense, behaviour of some other s.d.s. $S_{2}$, whose states (or some information about them) $\mathbf{E}$ are given. The main problem, to be formulated more precisely below, is to determine Markovian representations $\mathbf{G}$ (as a state space of an s.d.s. $S_{1}$ ) for a family $\mathbf{H}$ such that family $\mathbf{G}$ provides exactly the same amount of information about the family $\mathbf{H}$ as family $\mathbf{E}$ (see Definition 2.10).

It is clear that all the results of this paper can be extended on the $\sigma$-algebras generated by finite-dimensional Gaussian random variables. But, in the case that $\sigma$-algebras are arbitrary, the extensions of the proofs of this paper are nontrivial because one cannot take an orthogonal complement with respect to a $\sigma$-algebra as one can with respect to subspaces in a Hilbert space. 


\section{Preliminary notions and definitions}

Let $\mathbf{F}=\left(F_{t}\right), t \in \mathbb{R}$, be a family of Hilbert spaces. We will think about $F_{t}$ as the information available at time $t$, or as a current information. Total information $F_{<\infty}$ carried by $\mathbf{F}$ is defined by $F_{<\infty}=\vee_{t \in \mathbb{R}} F_{t}$, while past and future information of $\mathbf{F}$ at $t$ is defined as $F_{\leq t}=$ $\vee_{s \leq t} F_{s}$ and $F_{\geq t}=\vee_{s \geq t} F_{s}$, respectively. It is to be understood that $F_{<t}=\vee_{s<t} F_{s}$ and $F_{>t}=$ $\vee_{s>t} F_{s}$ do not have to coincide with $F_{\leq t}$ and $F_{\geq t}$, respectively; $F_{<t}$ and $F_{>t}$ are sometimes referred to as the real past and real future of $\mathbf{F}$ at $t$. Analogous notation will be used for families $\mathbf{H}=\left(H_{t}\right), \mathbf{G}=\left(G_{t}\right), \mathbf{E}=\left(E_{t}\right)$, and $\mathbf{J}=\left(J_{t}\right)$.

If $F_{1}$ and $F_{2}$ are arbitrary subspaces of a Hilbert space $\mathscr{H}$, then $P\left(F_{1} \mid F_{2}\right)$ will denote the orthogonal projection of $F_{1}$ onto $F_{2}$ and $F_{1} \ominus F_{2}$ will denote a Hilbert space generated by all elements $x-P\left(x \mid F_{2}\right)$, where $x \in F_{1}$. If $F_{2} \subseteq F_{1}$, then $F_{1} \ominus F_{2}$ coincides with $F_{1} \cap F_{2}^{\perp}$, where $F_{2}^{\perp}$ is the orthogonal complement of $F_{2}$ in $\mathcal{H}$.

Possibly the weakest form of causality can be introduced in the following way.

Definition 2.1. It is said that $\mathbf{H}$ is submitted to $\mathbf{G}$ (and written as $\mathbf{H} \subseteq \mathbf{G}$ ) if $H_{\leq t} \subseteq G_{\leq t}$ for each $t$.

It will be said that families $\mathbf{H}$ and $\mathbf{G}$ are equivalent (and written as $\mathbf{H}=\mathbf{G}$ ) if $\mathbf{H} \subseteq \mathbf{G}$ and $\mathbf{G} \subseteq \mathbf{H}$.

Definition 2.2. It is said that $\mathbf{H}$ is strictly submitted to $\mathbf{G}$ (and written as $\mathbf{H} \leq \mathbf{G}$ ) if $H_{t} \subseteq G_{t}$ for each $t$.

It is easy to see that strict submission implies submission and that the converse does not hold.

The notions of minimality and maximality of families of Hilbert spaces are specified in the following definition.

Definition 2.3. It will be said that $\mathbf{F}$ is a minimal (resp., strictly minimal) family having a certain property if there is no family $\mathbf{F}^{*}$ having the same property which is submitted (resp., strictly submitted) to $\mathbf{F}$.

It will be said that $\mathbf{F}$ is a maximal (resp., strictly maximal) family having a certain property if there is no family $\mathbf{F}^{*}$ having the same property such that family $\mathbf{F}$ is submitted (resp., strictly submitted) to $\mathbf{F}^{*}$.

It should be understood that a minimal (resp., strictly minimal) and maximal (resp., strictly maximal) family having a certain property are not necessarily unique.

An important tool in definition of the Hilbert space s.d.s. is the concept of conditional orthogonality.

Definition 2.4 (compare with $[7,8]$ and conditional independence from [9]). If $F_{1}$ and $F_{2}$ are arbitrary Hilbert spaces, then it is said that $F$ is splitting for $F_{1}$ and $F_{2}$ or that $F_{1}$ and $F_{2}$ are conditionally orthogonal given $F$ (and written as $F_{1} \perp F_{2} \mid F$ ) if $F_{1} \ominus F \perp F_{2} \ominus F$. When $F$ is trivial, that is, $F=\{0\}$, this reduces to the usual orthogonality $F_{1} \perp F_{2}$.

The following result gives an alternative way of defining splitting.

Lemma 2.5 (see $[1,9]) . F_{1} \perp F_{2} \mid F$ if and only if $P\left(F_{i} \mid F_{j} \vee F\right) \subseteq F$ for $i, j=1,2, i \neq j$. 
The following results concerning splitting will be used later (for the proof, see the given reference).

Theorem 2.6 (see [3]). The space $F$ is a minimal one such that $F_{1} \perp F_{2} \mid F$ if and only if $F=P\left(F_{1} \mid S\right)$ for some space $S$ such that $F_{2} \subseteq S \subseteq\left(F_{2} \vee P\left(F_{2} \mid F_{1}\right)\right) \oplus\left(F_{1} \vee F_{2}\right)^{\perp}$.

Corollary 2.7 (see [3]). The space $F \subseteq F_{1} \vee F_{2}$ is a minimal one such that $F_{1} \perp F_{2} \mid F$ if and only if $F=P\left(F_{1} \mid S\right)$ for some space $S$ such that $F_{2} \subseteq S \subseteq F_{2} \vee P\left(F_{2} \mid F_{1}\right)$.

In this paper, the following definition of Markovian property will be used.

Definition 2.8 (compare with $[7,8]$ ). Family $\mathbf{G}$ will be called Markovian if $P\left(G_{\geq t} \mid G_{\leq t}\right)=$ $G_{t}$ for each $t$.

Now we give a definition of an s.d.s. in terms of Hilbert spaces using conditional orthogonality relation. The characterizing property is the condition that past information of outputs and states and future information of outputs and states are conditionally orthogonal given the current state.

Definition 2.9 (see [3] and compare with [9]). An s.d.s. is a set of two families, $\mathbf{H}$ (outputs) and $\mathbf{G}$ (states), that satisfy the condition

$$
H_{<t} \vee G_{<t} \perp H_{>t} \vee G_{>t} \mid G_{t}
$$

For given family of outputs $\mathbf{H}$, any family $\mathbf{G}$ satisfying (2.1) is called a realization of an s.d.s. with those outputs.

It is clear that the realization of an s.d.s. is Markovian.

The next intuitively justifiable notion of causality has been proposed in [5].

Definition 2.10. It is said that $\mathbf{G}$ is a cause of $\mathbf{E}$ within $\mathbf{H}$ (and written as $\mathbf{E} \mid<\mathbf{G} ; \mathbf{H}$ ) if $E_{<\infty} \subseteq H_{\leq \infty}, \mathbf{G} \subseteq \mathbf{H}$ and $E_{<\infty} \perp H_{\leq t} \mid G_{\leq t}$ for each $t$.

Intuitively, $\mathbf{E}<\mathbf{G} ; \mathbf{H}$ means that, for arbitrary $t$, information about $E_{<\infty}$ provided by $H_{\leq t}$ is not "bigger" than that provided by $G_{\leq t}$. The meaning of this interpretation is specified in the next result.

Lemma 2.11 (see [1]). $\mathbf{E} \mid<\mathbf{G} ; \mathbf{H}$ if and only if $E_{<\infty} \subseteq H_{<\infty}, \mathbf{G} \subseteq \mathbf{H}$, and $P\left(E_{<\infty} \mid H_{\leq t}\right)=$ $P\left(E_{<\infty} \mid G_{\leq t}\right)$ for each $t$.

A definition, analogous to Definition 2.10, formulated in terms of $\sigma$-algebras, was first given in [4]; however, a strict Hilbert space version of the definition in [4] contains also the condition $\mathbf{E} \subseteq \mathbf{H}$ (instead of $E_{<\infty} \subseteq H_{<\infty}$ ) which does not have intuitive justification.

If $\mathbf{G}$ and $\mathbf{H}$ are such that $\mathbf{G} K \mathbf{G} ; \mathbf{H}$, we will say that $\mathbf{G}$ is its own cause within $\mathbf{H}$ (compare with [4]). It should be mentioned that the notion of subordination (as introduced in $[7,8]$ ) is equivalent to the notion of being one's own cause, as defined here.

If $\mathbf{G}$ and $\mathbf{H}$ are such that $\mathbf{G}<\mathbf{G} ; \mathbf{G} \vee \mathbf{H}$ (where $\mathbf{G} \vee \mathbf{H}$ is a family determined by $(G \vee$ $H)_{t}=G_{t} \vee H_{t}$ ), we will say that $\mathbf{H}$ does not cause $\mathbf{G}$. It is clear that the interpretation of Granger causality is now that $\mathbf{H}$ does not cause $\mathbf{G}$ if $\mathbf{G} \mid<\mathbf{G} ; \mathbf{G} \vee \mathbf{H}$ (see [4]). Without 
difficulty, it can be shown that this term and the term "H does not anticipate G" (as introduced in $[7,8]$ ) are identical.

\section{Causality and stochastic dynamic systems}

Suppose that an s.d.s. $\mathbf{S}_{1}$ causes, in a certain sense, behaviour of some other s.d.s. $\mathbf{S}_{2}$. It is natural to assume that outputs $\mathbf{H}$ of system $\boldsymbol{S}_{1}$ can be registered and that some information $\mathbf{E}$ about the states (or perhaps states themselves) of system $\mathbf{S}_{2}$ is given. Results that we will prove will tell us under which conditions concerning the relationships between $\mathbf{H}$ and $\mathbf{E}$ it is possible to find states $\mathbf{G}$ of system $\boldsymbol{S}_{1}$ which are in a certain causality relationship in the sense of Definition 2.10 with $\mathbf{H}$ and $\mathbf{E}$. More precisely, the following two cases will be considered:

(i) states of an s.d.s. $S_{1}$ are a cause of outputs of the same system within available information about s.d.s. $\mathbf{S}_{2}$;

(ii) the available information about $S_{2}$ is a cause of outputs of $S_{1}$ within states of $S_{1}$.

This paper is a continuation of the papers $[1,5,6]$.

We consider different kinds of causality between families $\mathbf{G}, \mathbf{H}$, and $\mathbf{E}$, while $\mathbf{G}$ and $\mathbf{H}$ are in the same relationship, that is, $\mathbf{G}$ is a realization of an s.d.s. with outputs $\mathbf{H}$ in all cases.

The following results give the solutions of the problem (i).

Theorem 3.1. Let $\mathbf{H}$ and $\mathbf{E}$ be such that $P\left(E_{t} \mid H_{<\infty}\right) \subseteq E_{\leq t}$ and $P\left(E_{<t} \mid H_{<\infty}\right) \perp$ $H_{>t} \mid P\left(E_{t} \mid H_{<\infty}\right)$ for each $t$. If the family $\mathbf{E}$ is Markovian, then the family $\mathbf{G}$, defined by

$$
G_{t}=P\left(E_{t} \mid H_{<\infty}\right), \quad t \in \mathbb{R},
$$

is a minimal realization (of an s.d.s. with outputs $\mathbf{H}$ ) that causes $\mathbf{H}$ within $\mathbf{E}$.

Proof. From $G_{\leq t}=P\left(E_{\leq t} \mid H_{<\infty}\right)$, it follows that $H_{<\infty} \perp E_{\leq t} \mid G_{\leq t}$. Also, the definition of $\mathbf{G}$ and the assumption $P\left(E_{t} \mid H_{<\infty}\right) \subseteq E_{\leq t}$ imply $G_{\leq t} \subseteq E_{\leq t}$, which together with the previous orthogonality relation means $\mathbf{H} \mid<\mathbf{G}$; $\mathbf{E}$. The minimality of $\mathbf{G}$ follows from Theorem 2.6 and Corollary 2.7.

From $G_{\leq t} \subseteq E_{\leq t}$, the fact that $P\left(G_{\geq t} \mid G_{\leq t}\right)=P\left(E_{\geq t} \mid G_{\leq t}\right)$ which follows from $G_{<\infty}=$ $H_{<\infty},(3.1)$ and the assumption that $\mathbf{E}$ is Markovian, we get

$$
P\left(G_{\geq t} \mid G_{\leq t}\right)=P\left(E_{\geq t} \mid G_{\leq t}\right)=P\left(P\left(E_{\geq t} \mid E_{\leq t}\right) \mid G_{\leq t}\right)=P\left(E_{t} \mid G_{\leq t}\right) .
$$

The relation $\mathbf{H} K \mathbf{G}$; E (because $H_{<\infty}=G_{<\infty}$ ) particularly implies $G_{<\infty} \ominus G_{\leq t} \perp E_{t}$, so that (3.2) becomes

$$
P\left(G_{\geq t} \mid G_{\leq t}\right)=P\left(E_{t} \mid G_{<\infty}\right)=P\left(E_{t} \mid H_{<\infty}\right)=G_{t},
$$

which means that $\mathbf{G}$ is Markovian. This fact with the assumption $G_{<t} \perp H_{>t} \mid G_{t}$ gives $G_{\leq t} \perp H_{>t} \vee G_{\geq t} \mid G_{t}$. However, since $H_{<t} \subseteq G_{\leq t}$ (which is an obvious consequence of $\mathbf{H} K \mathbf{G} ; \mathbf{E}$ ), the last relation implies that $\mathbf{G}$ is a realization of an s.d.s. with outputs $\mathbf{H}$.

If $\mathbf{H}$ is its own cause within $\mathbf{E}$, we obtain a simpler version of the last result. 
Corollary 3.2. Let $\mathbf{H}$ be its own cause within $\mathbf{E}$ and $P\left(E_{<t} \mid H_{\leq t}\right) \perp H_{>t} \mid P\left(E_{t} \mid H_{\leq t}\right)$ for each $t$. If $\mathbf{E}$ is Markovian, then the family $\mathbf{G}$, defined by

$$
G_{t}=P\left(E_{t} \mid H_{\leq t}\right), \quad t \in \mathbb{R},
$$

is a minimal realization (of an s.d.s. with outputs $\mathbf{H}$ ) that causes $\mathbf{H}$ within $\mathbf{E}$.

The following result does not require $\mathbf{E}$ to be Markovian but provides a realization whose present information at $t$ is equal to its total information accumulated up to $t$.

Theorem 3.3. Let $\mathbf{H}$ and $\mathbf{E}$ be such that $H_{<\infty} \subseteq E_{<\infty}$ and $P\left(E_{t} \mid S\right) \subseteq E_{\leq t}$ for each $t$ where $S$ is some space such that $H_{<\infty} \subseteq S \subseteq H_{<\infty} \vee P\left(H_{<\infty} \mid E_{\leq t}\right)$. Family $\mathbf{G}$ is a minimal realization (of an s.d.s. with outputs $\mathbf{H}$ ) that causes $\mathbf{H}$ within $\mathbf{E}$ if and only if it is defined by

$$
G_{t}=P\left(E_{\leq t} \mid S\right), \quad t \in \mathbb{R} .
$$

Proof. Let (3.5) hold. From Theorem 2.6 and Corollary 2.7, it follows that $G_{t}$ is a minimal space such that $H_{<\infty} \perp E_{\leq t} \mid G_{t}$ if and only if it is defined by (3.5). Since $G_{t}=G_{\leq t}$, it follows that $H_{<\infty} \perp E_{\leq t} \mid G_{\leq t}$, which because $\mathbf{G} \subseteq \mathbf{E}$ (it follows from the assumption $P\left(E_{t} \mid S\right) \subseteq E_{<t}$ and (3.5)) is equivalent to $\mathbf{H}<\mathbf{G}$; $\mathbf{E}$.

From $\mathbf{H} \subseteq \mathbf{G}$ (it is clear from (3.5)) and $G_{t}=G_{\leq t}$, it follows immediately that $\mathbf{G}$ is a realization of an s.d.s. with outputs $\mathbf{H}$.

The converse is trivial.

The next example illustrates the above result.

Example 3.4. Let $Z(t)=\sum_{n=1}^{2} \int_{0}^{t} g_{n}(t, u) d Z_{n}(u), t \in[0,1]$, be a proper canonical representation of the process $\{Z(t), t \in[0,1]\}$ (see $[7,8]$ ) and let the process $\{X(t), t \in[0,1]\}$ be defined by $X(t)=\int_{0}^{t} h(u) d Z_{1}(u), t \in[0,1]$. Then $F_{\leq t}^{X} \subseteq F_{\leq t}^{Z}$ for each $t$. Further, for $Y(t)=P\left(Z(t) \mid F_{<\infty}^{X}\right)=\int_{0}^{1} g_{1}(t, u) d Z_{1}(u), t \in[0,1]$, we have that $F_{\leq t}^{Y} \subseteq F_{\leq t}^{Z}$. According to Theorem 3.3 (for $\mathbf{H}=\mathbf{F}^{X}=\left(F_{t}^{X}\right), t \in[0,1], F_{t}^{X}=\{c \cdot X(t), c \in \mathbb{R}\}, \mathbf{E}=\mathbf{F}^{Z}$ and $\mathbf{G}_{t}=\mathbf{F}^{Y}$ ), it follows that family $F_{t}^{Y}=P\left(F_{\leq t}^{Z} \mid F_{<\infty}^{X}\right), t \in[0,1]$, is a minimal realization (of an s.d.s. with outputs $\left.F_{\leq t}^{X}\right)$ that causes $F_{\leq t}^{X}$ within $Z(t)$.

In the remaining part of the paper, we consider the problem (ii) formulated above.

In part (i), we considered realizations $\mathbf{G}$ such that $\mathbf{G} \subseteq \mathbf{E}$, that is, the given family $\mathbf{E}$ is a natural "framework" in which we find realizations G of an s.d.s. $S_{1}$. However, in the case (ii), where $\mathbf{E} \subseteq \mathbf{G}$, the family $\mathbf{E}$ is submitted to unknown family $\mathbf{G}$, so that we will assume that all considered families of Hilbert spaces are submitted to some given "framework" family $\mathbf{F}$ of Hilbert spaces.

The following theorem considers the problem of determining the possible states $\mathbf{G}$ (of an s.d.s. with outputs $\mathbf{H}$ ) such that the family $\mathbf{E}$ is a cause of outputs $\mathbf{H}$ within $\mathbf{G}$.

Theorem 3.5. (1) Let $\mathbf{H}$ and $\mathbf{E}$ be such that $\mathbf{H} \subseteq \mathbf{E}$. Each Markovian family $\mathbf{G}$ such that $\mathbf{E}<\mathbf{E} ; \mathbf{G}$ and $G_{<t} \perp H_{>t} \mid G_{t}$ for each $t$ is a realization (of an s.d.s. with outputs $\mathbf{H}$ ) and $\mathbf{E}$ is a cause of $\mathbf{H}$ within $\mathbf{G}$. 
(2) Let $\mathbf{H}, \mathbf{E}$, and $\mathbf{J}$ be such that $\mathbf{H} \subseteq \mathbf{E}$, as well as $\mathbf{E} K \mathbf{E} ; \mathbf{J}$ and $P\left(J_{<t} \mid E_{\leq t}\right) \perp H_{>t} \mid P\left(J_{t} \mid E_{\leq t}\right)$ for each $t$. If $\mathbf{J}$ is Markovian, then the family $\mathbf{G}$, defined by

$$
G_{t}=P\left(J_{t} \mid E_{\leq t}\right), \quad t \in \mathbb{R},
$$

is minimal among the realizations (of an s.d.s. with outputs $\mathbf{H}$ ) such that $\mathbf{E}$ is a cause of $\mathbf{H}$ within $\mathbf{G}$.

(3) If $\mathbf{H} \subseteq \mathbf{E}$ and if given "framework" family $\mathbf{F}$ such that $\mathbf{E}<\mathbf{E} ; \mathbf{F}$, then the family $\mathbf{G}$, defined by

$$
G_{t}=F_{\leq t}, \quad t \in \mathbb{R},
$$

is strictly maximal among the realizations (of an s.d.s. with outputs $\mathbf{H}$ ) such that $\mathbf{E}$ is a cause of $\mathbf{H}$ within $\mathbf{G}$.

Proof. (1) According to Lemma 2.5, the assumption $G_{<t} \perp H_{>t} \mid G_{t}$ is equivalent to $P\left(H_{>t} \mid G_{\leq t}\right) \subseteq G_{t}$. From that and the assumption that $\mathbf{G}$ is Markovian family, we get

$$
G_{t}=P\left(G_{\geq t} \mid G_{\leq t}\right)=P\left(G_{\geq t} \vee H_{>t} \mid G_{\leq t}\right)
$$

which is equivalent to $G_{\leq t} \perp G_{\geq t} \vee H_{>t} \mid G_{t}$. However, since $H_{<t} \subseteq G_{\leq t}$ (which is an obvious consequence of $\mathbf{H} \subseteq \mathbf{E}$ and $\mathbf{E}<\mathbf{E} ; \mathbf{G}$ ), the last relation means that $\mathbf{G}$ is a realization of an s.d.s. with outputs $\mathbf{H}$. From $\mathbf{E}<\mathbf{E} ; \mathbf{G}$ and $\mathbf{H} \subseteq \mathbf{E}$, it follows that $\mathbf{H}<\mathbf{E}$; $\mathbf{G}$ holds.

(2) From (3.6), it follows that $G_{\leq t}=E_{\leq t}$ and immediately we get $\mathbf{H}<\mathbf{E}$; $\mathbf{G}$. According to Definition 2.10, it is clear that the family $\mathbf{G}$, defined by (3.6), is a minimal family such that $\mathbf{H} \mid<\mathbf{E} ; \mathbf{G}$. From the assumptions that $\mathbf{E} \mid<\mathbf{E} ; \mathbf{J}$ and the fact that $\mathbf{J}$ is Markovian, we get $P\left(G_{\geq t} \mid G_{\leq t}\right)=P\left(J_{\geq t} \mid E_{\leq t}\right)=P\left(P\left(J_{\geq t} \mid J_{\leq t}\right) \mid E_{\leq t}\right)=P\left(J_{t} \mid E_{\leq t}\right)=G_{t}$ which means that $\mathbf{G}$ is Markovian. Now, according to part (1) of this theorem, it follows that the family $\mathbf{G}$, defined by (3.6), is a realization (of an s.d.s. with outputs $\mathbf{H}$ ) such that $\mathbf{H}<\mathbf{E}$; $\mathbf{G}$.

(3) Since $G_{\leq t}=F_{\leq t}$, the assumption $\mathbf{E}<\mathbf{E} ; \mathbf{F}$ is equivalent to $\mathbf{E}<\mathbf{E} ; \mathbf{G}$, so that because of $\mathbf{H} \subseteq \mathbf{E}$, it follows that $\mathbf{H} K \mathbf{E} ; \mathbf{G}$. From $G_{t}=G_{\leq t}$ and $\mathbf{H} \subseteq \mathbf{G}$, it immediately follows that $\mathbf{G}$ is a realization of an s.d.s. with outputs $\mathbf{H}$. From the fact that $\mathbf{F}$ is a "framework" family (i.e., $\mathbf{G} \subseteq \mathbf{F}$ ), it is clear that $\mathbf{G}$ is a strictly maximal realization with given properties.

It is easy to see that for given outputs $\mathbf{H}$ of an s.d.s. $S_{1}$ and information $\mathbf{E}$ about an s.d.s. $S_{2}$, the family $\mathbf{G}$, defined by (3.6), is not a unique minimal realization (of an s.d.s. $S_{1}$ ) such that $\mathbf{H} \mid<\mathbf{E} ; \mathbf{G}$. For each family $\mathbf{J}^{*} \subseteq \mathbf{F}$ which satisfies conditions from Theorem 3.5(2), with $G_{t}^{*}=P\left(J_{t}^{*} \mid E_{\leq t}\right), t \in \mathbb{R}$, is defined a minimal realization (of an s.d.s. $S_{1}$ ) such that $\mathbf{H} K \mathbf{E} ; \mathbf{G}^{*}$. All these minimal realizations have the past information equivalent to $E_{\leq t}$, $t \in \mathbb{R}$, but their present information at $t$ is different.

The next example shows that family G defined by (3.6) is not strictly minimal realization of an s.d.s. with outputs $\mathbf{H}$ such that $\mathbf{H} K \mathbf{E} ; \mathbf{G}$. 
Example 3.6. Let $A$ and $B$ be arbitrary Hilbert spaces and let $\mathbf{H}=\left(H_{t}\right), \mathbf{E}=\left(E_{t}\right)$, and $\mathbf{J}=\left(J_{t}\right), t \in\{1,2,3\}$, be defined by

$$
\begin{aligned}
& H_{1}=A, \quad H_{2}=B, \quad H_{3}=A \vee B, \\
& E_{1}=A, \quad E_{2}=B, \quad E_{3}=A, \\
& J_{1}=A, \quad J_{2}=A \vee B, \quad J_{3}=B .
\end{aligned}
$$

It is easy to see that $\mathbf{J}$ is Markovian, $\mathbf{E} K \mathbf{E}$; J and $P\left(J_{<t} \mid E_{\leq t}\right) \perp H_{>t} \mid P\left(J_{t} \mid E_{\leq t}\right)$ for each $t$. If the family $\mathbf{G}$ is defined by (3.6), then

$$
G_{1}=A, \quad G_{2}=A \vee B, \quad G_{3}=B
$$

According to Theorem 3.5(2), $\mathbf{G}$ is a minimal realization (of an s.d.s. with outputs $\mathbf{H}$ ) and $\mathbf{H} K \mathbf{E} ; \mathbf{G}$. However, the family $\mathbf{G}^{*}=\left(G_{t}^{*}\right), t \in\{1,2,3\}$, defined by

$$
G_{1}^{*}=A, \quad G_{2}^{*}=A \vee B, \quad G_{3}^{*}=\{0\},
$$

is another realization of the same s.d.s. and $\mathbf{H}<\mathbf{E} ; \mathbf{G}^{*}$. Obviously, $\mathbf{G}^{*} \leq \mathbf{G}$.

The problem of determining a strictly minimal realization $\mathbf{G}$ (of an s.d.s. with outputs $\mathbf{H})$ such that $\mathbf{H}<\mathbf{E} ; \mathbf{G}$ is still open. If it would be possible to find a strictly minimal family $\mathbf{J}^{m}$ between families $\mathbf{J}^{*} \subseteq \mathbf{F}$ that satisfy conditions from Theorem 3.5(2), this strictly minimal family $\mathbf{J}^{m}$ would define a strictly minimal family $\mathbf{G}^{m}$ (with (3.6)) among all families $\mathrm{G}$ of Theorem 3.5(2). It is clear that if there exists such strictly minimal family, it cannot be necessarily unique, so that a strictly minimal realization with given properties is not necessarily unique.

Remark 3.7. It is of interest to find conditions for the existence of realizations with certain properties less restrictive than those obtained in this paper.

\section{Acknowledgment}

This research was supported by the Science Fund of Serbia, through the Mathematical Institute, Belgrade.

\section{References}

[1] J. B. Gill and Lj. Petrović, Causality and stochastic dynamic systems, SIAM J. Appl. Math. 47 (1987), no. 6, 1361-1366.

[2] C. W. J. Granger, Investigating causal relations by econometric models and cross-spectral methods, Econometrica 37 (1969), 424-438.

[3] A. Lindquist, G. Picci, and G. Ruckebusch, On minimal splitting subspaces and Markovian representations, Math. Systems Theory 12 (1978/1979), no. 3, 271-279.

[4] P. A. Mykland, Statistical causality, Statistical Report no. 14, Department of Mathematics, University of Bergen, 1986.

[5] Lj. Petrović, Causality and stochastic realization problem, Publ. Inst. Math. (Beograd) (N.S.) 45(59) (1989), 203-210.

[6] Causality and Markovian reductions and extensions of a family of Hilbert spaces, J. Math. Systems Estim. Control 8 (1998), no. 4, 12 pp. 


\section{Causality and stochastic realization}

[7] Yu. A. Rozanov, Theory of Innovation Processes, Monographs in Probability Theory and Mathematical Statistics, Izdat. Nauka, Moscow, 1974.

[8] Innovation Processes, V. H. Winston \& Sons, New York, 1977.

[9] C. van Putten and J. H. van Schuppen, On stochastic dynamical systems, Proc. 4th International Symposium on the Mathematical Theory of Networks and Systems, vol. 3, Western Periodicals, California, 1979, pp. 350-355.

Ljiljana Petrović: Faculty of Economics, Belgrade University, 11000 Belgrade, Serbia and Montenegro

E-mail address: petrovl@one.ekof.bg.ac.yu 


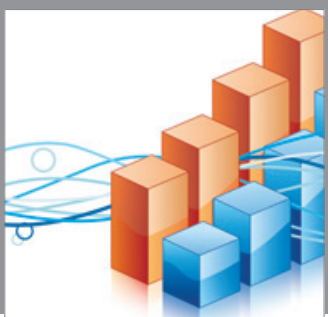

Advances in

Operations Research

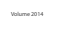

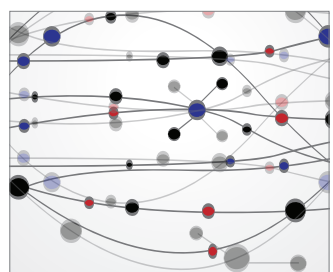

\section{The Scientific} World Journal
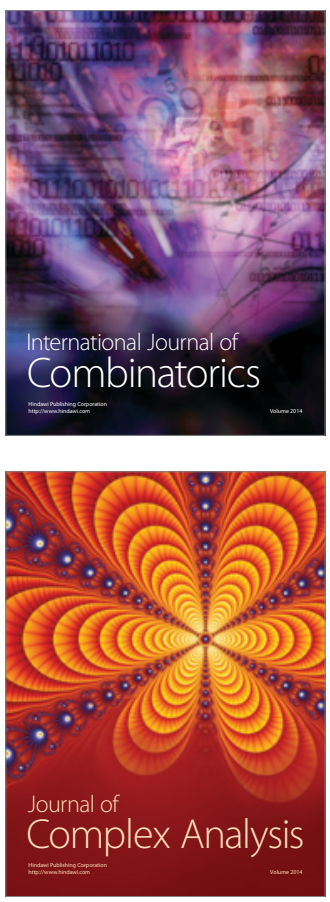

International Journal of

Mathematics and

Mathematical

Sciences
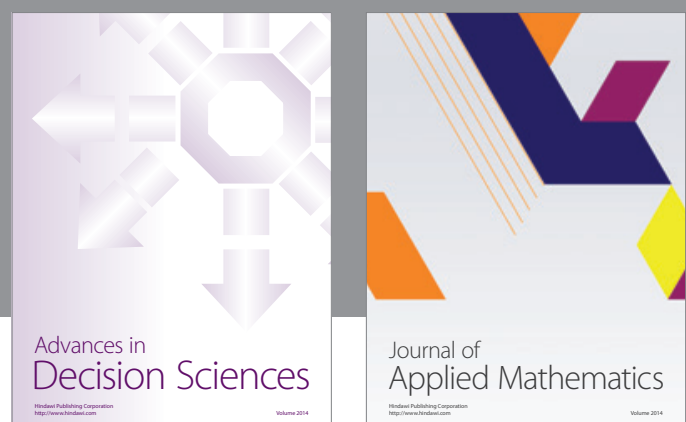

Journal of

Applied Mathematics
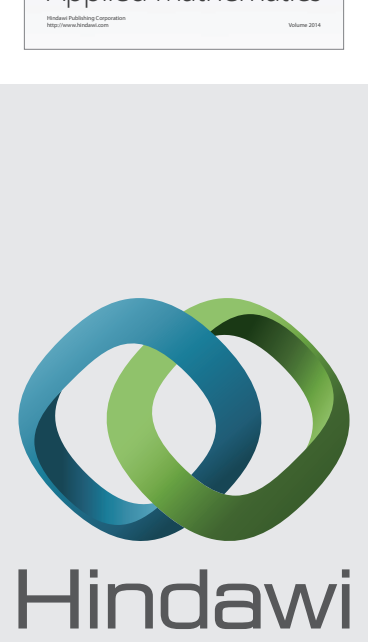

Submit your manuscripts at http://www.hindawi.com
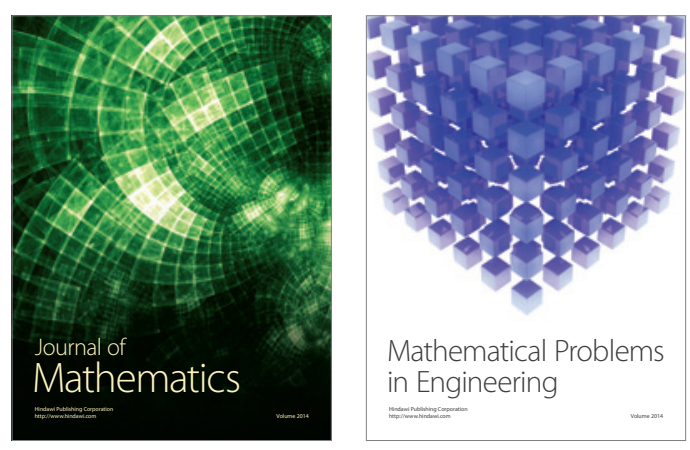

Mathematical Problems in Engineering
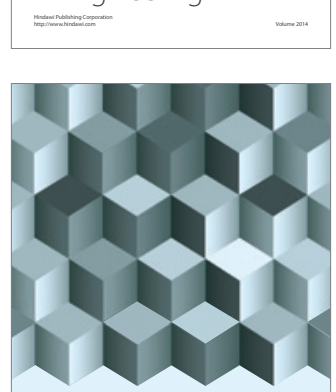

Journal of

Function Spaces
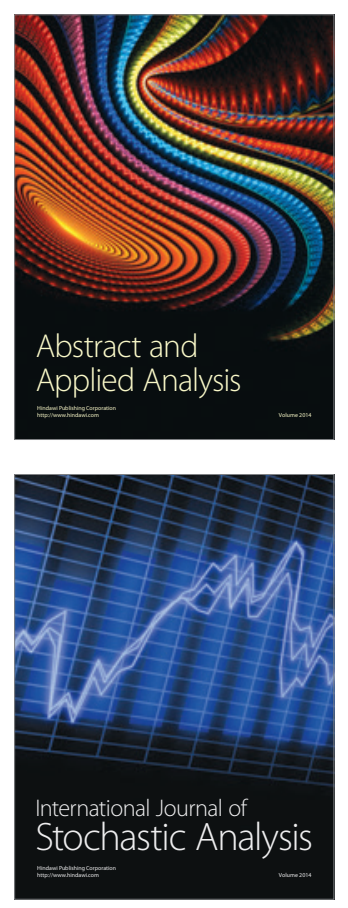

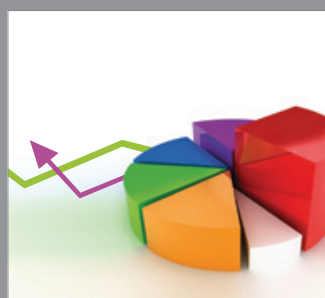

ournal of

Probability and Statistics

Promensencen
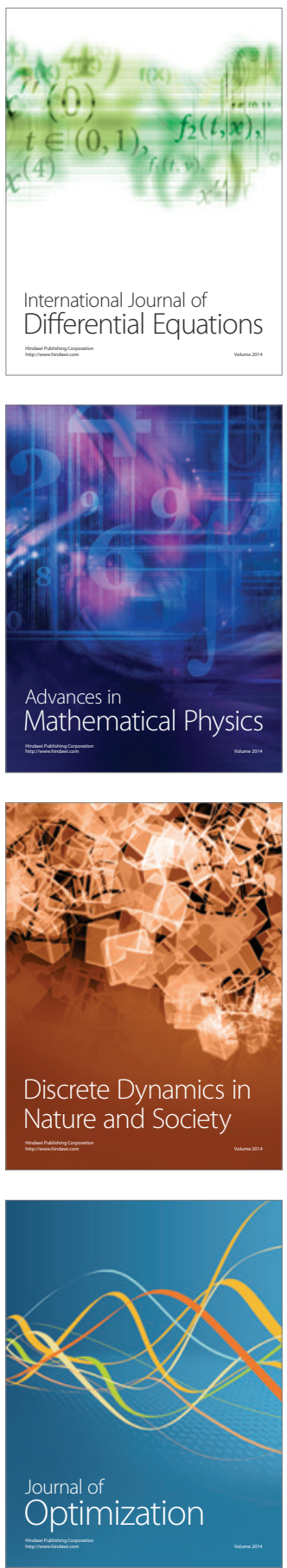